\title{
Parâmetros genéticos da curva de produção de ovos de uma linha fêmea de frango de corte
}

\author{
Evaluation of posture rating curves of a female line of broilers
}

\author{
Valdecy Aparecida Rocha Cruz ${ }^{*}$ Aldrin Vieira Pires ${ }^{\mathrm{I}}$ Rodolpho Almeida Torres Filho $^{\mathrm{II}}$ \\ Idalmo Garcia Pereira ${ }^{I}$ Claudio Vieira Araujo ${ }^{\text {III }}$ Camila Tangari Meira
}

\section{- NOTA-}

\section{RESUMO}

Objetivou-se com este trabalho estimar os parâmetros genéticos e fenotípicos dos parâmetros da curva de produção de ovos, pelo o modelo de polinômios segmentados ( " $x_{p}$ "- idade das aves ao pico de postura, " $P$ "- nível de produção no pico, "s" - taxa de decréscimo semanal na produção de ovos após o pico $e$ " $t$ " - tempo entre o início da postura $e$ o pico de produção de ovos). Foram utilizados dados da produção de ovos semanal da 25á até 64a semanas de idade, provenientes de 2.398 matrizes de uma linhagem de frangos de corte. A estimação dos componentes de covariâncias, herdabilidade e correlações para esses parâmetros da curva de postura foram obtidas por máxima verossimilhança restrita. As tendências genéticas foram estimadas com as médias dos valores genéticos dos parâmetros da curva em função do ano de nascimento das matrizes. As médias das herdabilidades foram de baixa a média magnitudes, 0,25 ( $\left.x_{p}\right), 0,18(P), 0,17$ (s) e 0,10 (t). As correlações genéticas entre o parâmetro t e os demais parâmetros da curva foram positivas, variando de 0,21 até 1,00, sendo menor entre te s e maior entre os parâmetros $x_{p}$ e t. Entre os parâmetros $x_{p}$ e $P$, a correlação também foi positiva, de magnitude média $(0,39)$. As correlações foram negativas entre os parâmetros $s$ e $x_{p}$ e s e $P$, apresentando-se menor entre s e $x_{p}(-0,17)$ e maior entre s e $P(-0,91)$. As tendências genéticas encontradas para os parâmetros indicaram uma redução no tempo entre o início e pico de produção e idade ao pico de postura. O nível de produção apresentou uma queda no valor genético ao longo do período avaliado. Pode-se observar possível ganho genético para os parâmetros da curva de produção de ovos, contudo, deve-se atentar para as correlações genéticas positivas entre os parâmetro $x_{p}$ e $P, o$ que pode levar a um aumento do nível de produção e a um aumento na idade ao pico de produção. $O$ mesmo caso acontece com os parâmetros $x_{p}$ es, indicando que as aves mais precoces ao pico poderão apresentar menor persistência.

Palavras-chave: curva de postura, polinômio segmentado, regressão não linear.

\section{ABSTRACT}

The objective of this study was to estimate genetic parameters and phenotypic parameters of egg production curve, using the segmented polynomial model( " $x_{p}$ " - the peak age of the birds in attitude, " $P$ " - at peak production level, "s" - rate of decline in weekly egg production after peak, and " $t$ " - time between the onset of lay and peak egg production). We used data from the weekly egg production from 25 to 64 weeks of age from 2,398 arrays of a lineage of broilers chickens. Estimation of covariance components, heritability and correlations for these parameters curve posture were obtained by restricted maximum likelihood. Genetic trends were estimated with the average breeding values the of parameters of the curve as a function of birth year of the arrays. The mean heritabilities were low to medium magnitude, 0.25 $\left(x_{p}\right), 0.18(P), 0.17(s)$ e $0.10(t)$. Genetic correlations among the parameter " $t$ " and the other parameters of the curve were positive ranging from 0.21 to 1.00 , being lower between " $t$ " and " $s$ ", and higher between the parameters $x_{p}$ and $t$. Between the parameters $x_{p}$ and $P$ the correlation was also positive, of an average magnitude (0.39). Correlations were negative between parameters " $s$ " and " $x_{p}$ ", and " $s$ " and " $P$ ", presenting itself lower between " $s$ " and " $x_{p}$ " (-0.17) and higher between " $s$ " and " $P$ " (-0.91). The genetic trends found for the parameters of the model indicated a reduction in time between the production's onset and peak, and age at production's peak. The level of production showed a decline in breeding value over

'Departamento de Zootecnia, Universidade Federal dos Vales do Jequitinhonha e Mucuri (UFVJM), 39100-000, Diamantina, MG, Brasil. E-mail: valdecya.r.cruz@gmail.com.*Autor para correspondência.

IIDepartamento de Zootecnia, Universidade Federal Fluminense (UFF), Niterói, RJ, Brasil.

"IIDepartamento de Zootecnia, Universidade Federal Mato do Grosso (UFMT), Sinop, MT, Brasil. 
the period evaluated. It can be observed a potential genetic gain for the egg's production parameters' curve; however, it must be paid special attention to the positive genetic correlations between the parameter $x_{p}$ and $P$. What can lead to an increase in the level of production and may lead to an increase in age at peak production. The same applies to the parameters $x_{p}$ indicating that birds with earlier peaks may present lower persistence.

Key words: curve posture, segmented polynomial, regression non linear.

Os parâmetros das curvas de produção de ovos, quando considerados como variáveis biológicas, podem fornecer informações que auxiliam um programa de melhoramento genético. Segundo LEDUR et al. (1993), as estimativas de parâmetros genéticos e fenotípicos são as principais ferramentas para se alcançar altos ganhos genéticos na população e se ter uma melhor seleção das linhagens.

Existem na literatura vários trabalhos sobre curva de produção de ovos, sendo que poucos (CONTI, 2007; ROSSI et al. 2010) estudaram os fatores genéticos relacionados com os parâmetros dos modelos. O conhecimento das correlações entre os parâmetros da curva de postura é importante ao se formularem programas de seleção.

O modelo de polinômios segmentados foi desenvolvido por FIALHO \& LEDUR (1997) e, segundo os autores, esse modelo foi testado contra outros modelos existentes, sendo constatado que possui maior poder de predição e igual ou melhor capacidade de ajuste. Além disso, estima parâmetros de fácil interpretação prática, o que o torna vantajoso na caracterização de curvas de produção. As características de cada curva podem ser descritas resumidamente pelos seus parâmetros e esses parâmetros podem ser comparados entre uma curva e outra, para determinar o efeito dos tratamentos na produção de ovos.

Com o objetivo de estudar os parâmetros genéticos e fenotípicos dos parâmetros da curva de produção de ovos por meio de modelos de polinômios segmentados, foram utilizados dados de 2.398 aves com registros semanais de uma linha fêmea de frangos de corte do pacote de genética da Globoaves, localizada no município de Catanduvas, SC. As informações referem-se à produção de ovo semanal das 25 as 64 semanas de idade, 2005 a 2006.

Os parâmetros da curva de produção foram estimados utilizando-se o modelo de polinômios segmentados (FIALHO \& LEDUR, 1997) em que: $Y=0$ para $x<x_{p}-t ; Y=P-3 . P\left(x_{p}-x\right) / t+2 . P\left(\left(x_{p}-x\right) / t\right)^{3}$ para $\mathrm{x}_{\mathrm{p}}-\mathrm{t} \leq \mathrm{x}<\mathrm{x}_{\mathrm{p}} \mathrm{e}, Y=P-s\left(x-x_{p}\right)$ para $x_{p} \leq x$, em que: $Y$ é a produção diária, em ovo/ave/dia; $x_{p}$ é a idade das aves, em semanas, no momento do pico de produção; $P$ é o nível de produção no pico, em ovo/ave/dia; $s$ é a taxa de decréscimo semanal na produção após o pico, em ovo/ave/semana; $t$ é o tempo entre o início da postura e o pico de produção de ovos, em semanas, e $x$ é a idade da ave em um determinado tempo, em semanas.

Foi ajustado o modelo de polinômios segmentados para a população e estimados os parâmetros da curva para cada indivíduo, utilizando o procedimento NLIN do SAS (2002). As estimativas dos componentes de (co)variâncias e dos parâmetros genéticos para os parâmetros da curva $\left(x_{p}, P, s\right.$ e $\left.t\right)$ foram obtidas pelo programa MTDFREML (BOLDMAN et al., 1995). As herdabilidades estimadas e valores genéticos preditos foram obtidos a partir desses componentes de variância, utilizando o seguinte modelo: $\quad \mathbf{y}=\mathbf{X} \underset{\sim}{\mathbf{b}}+\mathbf{Z} \mathbf{g}+\mathbf{e}$, em que: $\mathbf{y}=$ vetor de observaçõe $\tilde{e}\left(x_{p}, P\right.$, s e t); X = matriz de incidência de efeitos fixos; $\underset{\sim}{\mathbf{b}}=$ vetor de efeitos fixos (nascimento); $\mathrm{Z}=$ matriz de incidência de efeitos genéticos aditivos diretos; $\mathbf{g}$ = vetor de efeitos genéticos aditivos diretos; $\underset{\mathbf{e}}{\mathbf{e}}=$ vetor de efeito residual.

Foram realizadas análises bicaracterísticas para todos os parâmetros ( $\mathrm{x}_{\mathrm{p}}, \mathrm{P}, \mathrm{s}$ e t) do modelo ajustado. As tendências genéticas para os parâmetros da curva foram estimadas utilizando os valores genéticos médios anuais pelo procedimento REG do software do SAS.

As médias das estimativas das herdabilidades foram de baixa a média magnitude (Tabela 1), variando de 0,10 a 0,25, indicando que as condições ambientais e os efeitos dos fatores nãoaditivos têm grande influência sobre as características avaliadas. Esses resultados indicam que a seleção, considerando o parâmetro " $\mathrm{x}_{\mathrm{p}}$ ", teria uma melhor resposta que os demais, pois apresenta maior herdabilidade.

Tabela 1 - Estimativas das herdabilidades médias (diagonal) e correlações genéticas (acima da diagonal) e fenotípicas (abaixo da diagonal) da curva de produção de ovos.

\begin{tabular}{lcccc}
\hline Parâmetro & $\mathrm{x}_{\mathrm{p}}$ & $\mathrm{P}$ & $\mathrm{s}$ & $\mathrm{t}$ \\
\hline $\mathrm{X}_{\mathrm{p}}$ & 0,25 & 0,39 & $-0,17$ & 1,00 \\
$\mathrm{P}$ & 0,16 & 0,18 & $-0,91$ & 0,32 \\
$\mathrm{~S}$ & 0,11 & 0,22 & 0,17 & 0,21 \\
$\mathrm{t}$ & 0,56 & 0,01 & 0,12 & 0,10 \\
\hline
\end{tabular}

1 " $\mathrm{x}_{\mathrm{p}}$ "- idade das aves ao pico de postura, "P"- nível de produção no pico, "s" - taxa de decréscimo semanal na produção de ovos após o pico e "t" - tempo entre o início da postura e o pico de produção de ovos. 
Os valores das correlações genéticas (Tabela 1) estimadas entre os parâmetros da curva de postura foram de magnitude baixas a altas negativas e positivas. As correlações entre o parâmetro " $t$ " e os demais parâmetros da curva foram positivas, variando de 0,21 até 1,00 , sendo menor entre "t" e "s" e maior entre os parâmetros " $\mathrm{x}_{\mathrm{p}}$ " e "t". Essas correlações indicam que os animais com um maior tempo entre início e pico de postura $(\mathrm{t})$ tendem a apresentar uma maior idade ao pico de postura $\left(\mathrm{x}_{\mathrm{p}}\right)$, maior nível de produção(P) e maior queda de produção pós-pico (s). Entre os parâmetros " $x_{p}$ " e "P", a correlação também foi positiva, de magnitude média $(0,39)$. As correlações foram negativas entre os parâmetros "s" e " $x_{p}$ " e "s" e "P", apresentando-se menor entre " $s$ " $e$ " $x_{p}$ " $(-0,17)$ e maior entre s e P $(-0,91)$, indicando que um aumento no nível de produção e idade ao pico de produção acarretaria uma menor queda de produção de ovos pós-pico. Esses valores indicam que, se a seleção for praticada com a intenção de aumentar o valor genético para o parâmetro "P", haverá um aumento no valor genético para persistência de produção, como resposta correlacionada. Entretanto, as respostas correlacionadas entre "P" com outros parâmetros podem proporcionar maior idade ao pico de postura e maior intervalo entre início e pico de produção.
As tendências genéticas, estimadas pela regressão dos valores genéticos preditos médios sobre o período de tempo em que ocorreu o nascimento das fêmeas, 2005 a 2006, para os parâmetros da curva de produção (Figura 1) indicam progresso genético para o tempo entre o início e pico de postura e idade ao pico de postura, apresentando-se a uma taxa de variação média de $-0,012$ dias $a n o^{-1}$ e $-0,027$ dias ano ${ }^{-1}$, respectivamente. No entanto, o nível de produção ao pico e a persistência não tiveram os mesmos resultados, apresentando-se praticamente constantes ao longo do período avaliado, podendo ser trabalhado, na perspectiva de melhorar a persistência e o nível de produção ao pico de postura, visto que essas características são de grande importância na produção.

ROSSI et al. (2010) encontraram estimativas de herdabilidades para os parâmetros a e â na dieta com o nivel preconizado de energia, de 0,21 e 0,$50 ; 0,12$ e 0,$49 ;$ e 0,12 e 0,48 e, na dieta de baixa energia, 0,23 e 0,$50 ; 0,13$ e 0,$50 ;$ e 0,10 e 0,47 . Segundo os autores, para ambos os níveis de energia, as herdabilidades para o parâmetro $\beta$ foram altas, indicando que as linhagens analisadas, apresentam variações genéticas aditivas que permitem uma boa resposta à seleção. SILVA et al. (2004) avaliaram curva de postura de linhagens de aves

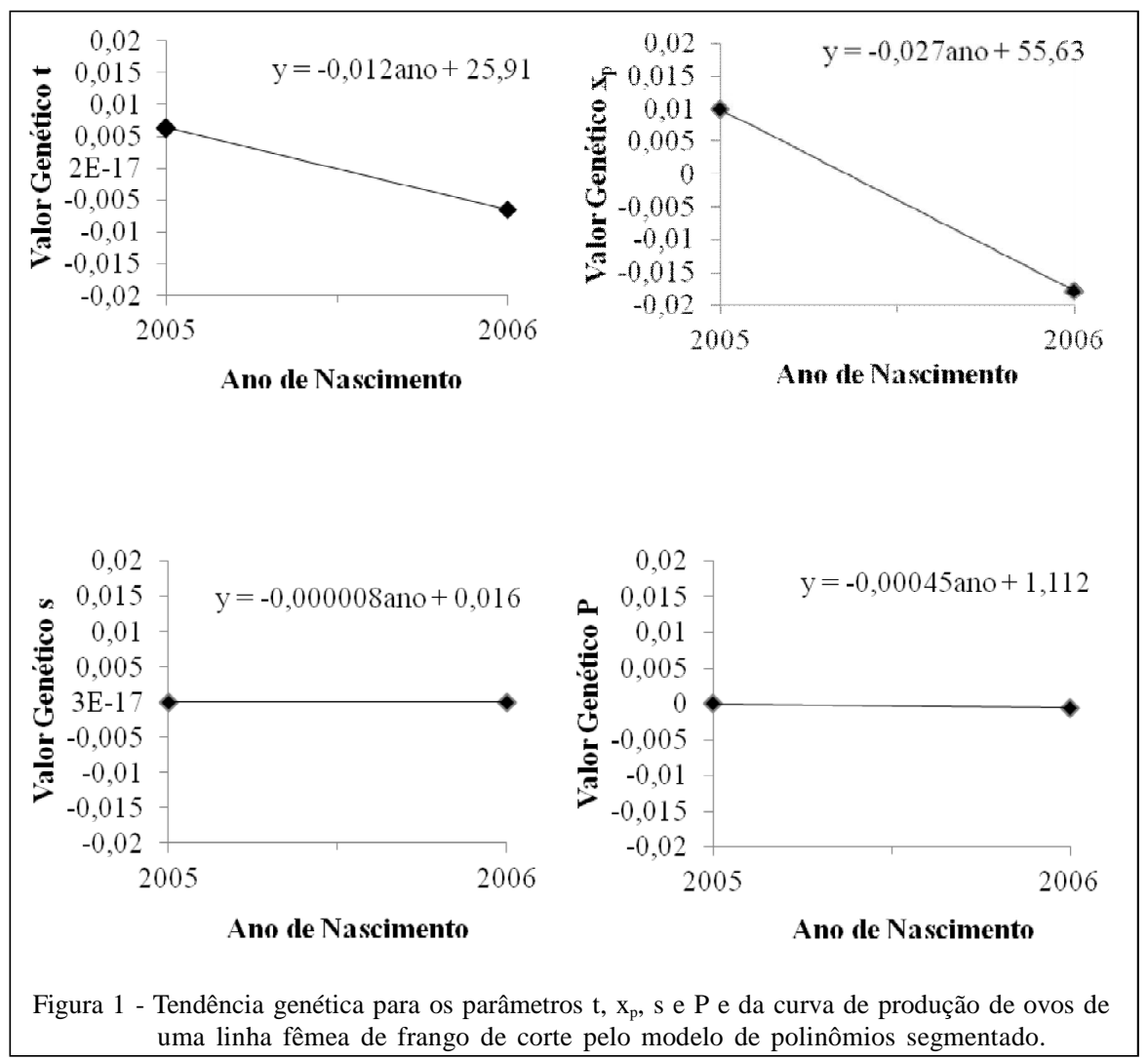

Ciência Rural, v.43, n.3, mar, 2013. 
White leghorn e relataram que o grupo selecionado para suportar maiores variações ambientais apresentou maior produção inicial de ovos, sendo, portanto, mais produtivo.

Os resultados do presente estudo sugerem que pode ser promovido ganho genético nos parâmetros da curva de produção de ovos, contudo, deve-se atentar para as correlações genéticas positivas entre os parâmetro $\mathrm{x}_{\mathrm{p}} \mathrm{e} \mathrm{P}$, pois a seleção para reduzir a idade das aves ao pico de postura $\left(\mathrm{x}_{\mathrm{p}}\right)$ levará à diminuição do valor genético e prejuízo no nível de produção no pico $(\mathrm{P})$. Assim, como nos parâmetros $\mathrm{x}_{\mathrm{p}} \mathrm{e}$ $\mathrm{s}$, a seleção para redução da idade ao pico de postura poderá levar uma menor persistência.

\section{AGRADECIMENTO}

Os autores agradecem à Globoaves, pela concessão dos dados para a realização deste trabalho.

\section{REFERÊNCIAS}

BOLDMAN, K.G. et al. A manual for use for MTDFREML. A set of programs to obtain estimates of variance and covariance [DRAFT]. Lincoln: Department of Agriculture, Agricultural Research Service, 1995. 120p.
CONTI, A.C.M. Análise genética da probabilidade diária de postura em três linhagens de codornas. 2007. 59. Dissertação (Mestrado em Zootecnia) - Universidade Estadual de Maringá, PR.

FIALHO, F.B.; LEDUR, M.C. Segmented polynomial model for estimation of egg production curves laying hens. British Poultry Science, v.1, n.38, p.66-73, 1997. Disponível em: <http://dx.doi.org/10.1080/00071669708417942>. Acesso em: 10 out. 2012. doi: 10.1080/00071669708417942

LEDUR, M.C. et al. Parâmetros genéticos e fenotípicos para características produtivas em linhagens de poedeiras de ovos brancos. Pesquisa Agropecuária Brasileira, v.28, n.9, p.1031-1037, 1993. Disponível em: <https://seer.sct.embrapa.br/ index.php/pab/article/view/3967/1258>.

ROSSI, R.M.; MARTINS, E.N Aspecto genéticos de curvas de probabilidade de postura em codornas. Revista Brasileira de Zootecnia, v.39, n.8, p.1708-1716, 2010. Disponível em: <http://www.scielo.br/scielo.php?script=sci_pdf\&pid=S1516$35982010000800012 \& \operatorname{lng}=$ en $\&$ nrm=iso\&tlng=pt $>$ Acesso em: 10 out. 2012. doi: 10.1590/S1516-35982010000800012.

SAS (STATISTICAL ANALYSIS SYSTEM). User's guide: statistics. Version 9.0, Carrey, 2002. 513p.

SILVA, F.F. et al. Análise Bayesiana da curva de postura de linhagens de aves White Leghorn. In: REUNIÃO ANUAL DA REGIÃO BRASILEIRA DA SOCIEDADE INTERNACIONAL DE BIOMETRIA, 49., 2004, Uberlândia, MG. Anais... Uberlândia: UFU, p. 328-332, 2004. 